\title{
IMAGING AND VISUALIZATION
}

\section{Natural animal magnetism}

A naturally occurring iron-sequestering protein is the key to a new strategy for visualizing gene expression with magnetic resonance imaging (MRI) that bypasses the of need for synthetic high-contrast agents.

MRI is a powerful diagnostic tool for clinical imaging, and many researchers have sought to develop MRI-based strategies that permit the high-resolution, real-time tracking of gene expression in living organisms. Several such techniques have emerged, most of which rely on the use of specially designed metal-complexed contrast compounds for labeling. These compounds, although effective in some cases, generally have drawbacks including poor penetrance into tissues. Carnegie Mellon University researcher Eric Ahrens has worked closely on this problem over the past several years, and sought an alternative solution that bypasses these obstacles.

"I'm very interested in magnetism," explains Ahrens, "and I became intrigued by these bacteria, magnetotactic bacteria, that make magnetic structures used for navigation." These unusual organisms migrate along geomagnetic lines with the assistance of magnetosomes, bacteriumproduced particles of crystallized magnetic material. "[Magnetosomes are] in many ways similar to superparamagnetic iron oxide particles," Ahrens continues, "which have gained wide interest for use in cellular imaging... I looked a little further and wondered if there were metalloproteins found in nature that also exhibited properties of superparamagnetism."

In the end, his group would focus on metalloproteins from the ferritin family, a widely studied class of proteins that are capable of storing iron molecules and have a vital role in regulating iron levels in the body. In a recent article in Nature Medicine, Ahrens' group describes the generation of an adenoviral construct for the constitutive expression of human ferritin. Initial in vitro experiments, wherein cultured cells were infected with the recombinant virus, demonstrated that ferritin was being produced, and that it was capable of effectively sequestering iron present in the culture medium. The resulting increase in iron-loaded ferritin appeared to have no negative impact on cell growth or viability, and allowed the cells to be readily visualized by MRI. Ahrens' group followed up with in vivo tests, injecting the virus into the brains of live mice, and found that within five days they could clearly visualize highly localized regions of strong MRI contrast at the site of injection. This labeling remained distinct even 39 days after injection.

The lab is now refining their system, but Ahrens ultimately sees this approach as a highly adaptable system for the precise in vivo analysis of transgene expression: "You could link this MRI reporter gene to any other gene of interest, including therapeutic genes for diseases like cancer and arthritis, to detect where and when they are being expressed."

\section{Michael Eisenstein}

\section{RESEARCH PAPERS}

Genove, G. et al. A new transgene reporter for in vivo magnetic resonance imaging. Nat. Med.,11, 450-454 (2005).

\section{GENOMICS}

\section{WHAT'S IN THE 'JUNK' OF THE GENOME?}

A large-scale analysis of the human transcriptome with unprecedented resolution raises the possibility that large numbers of transcripts are produced from regions of the genome considered as intergenic in the current annotation.

With the completion of sequencing the human genome and the availability of microarrays with ever-increasing density, one tends to grow accustomed to largescale genomic studies. But it was hard not to be taken aback by the ambitious nature of the study recently reported in Saience by a group at Affymetrix (Santa Clara, CA, USA), in which a team led by Thomas Gingeras has mapped the transcripts from ten human chromosomes at a five-nucleotide resolution.

This impressive resolution, meant to increase the likelihood of detecting short transcripts, was obtained by designing tiling arrays with 25-nucleotide probes spaced every five base pairs in non repeat sequence of each of ten chromosomes. Using 98 microarrays comprising some 74 million probe pairs, the team interrogated about $30 \%$ of the human genome. They analyzed cytosolic polyadenylated RNA from eight different cell lines and, for one cell line, extended the study to both poly $(A)+$ and $\operatorname{poly}(A)$-transcripts from both the cytosol and the nucleus.

Among several puzzling findings, the team concluded that whereas most of the detected cytosolic poly $(A)+$ transcripts map to genomic regions rich in exons and genes, about $30 \%$ of them seem to originate from currently unannotated regions. After analyzing the structure of a random subset of such transcripts by $5^{\prime}$ and $3^{\prime}$ RACE (rapid amplification of CDNA ends) and CDNA cloning, they also suggested that it is not unusual for both strands of DNA to be transcribed. Not the least of the surprises was the unexpected abundance of poly $(A)$-transcripts, and the authors estimated that these could comprise half of the human transcriptome and be similarly derived from both currently annotated and intergenic regions.

Many researchers agree that the current annotation of the human genome is most likely incomplete, and several recent reports have suggested the existence of more genes. With the present study, however, predictions of the extent of transcribed regions go beyond previous speculations. This provocative picture raises a lot of questions, and although microarray results are a starting point rather than a definitive answer, future investigation of the biogenesis and biological function of this bounty of new transcripts promises to yield exciting new developments.

\section{Veronique Kiermer}

\section{RESEARCH PAPERS}

Cheng, J. et al. Transcriptional maps of 10 human chromosomes at 5-nucleotide resolution. Science, published online 29 March 2005 (doi: 10.1126/ science.1108625). 